This is an electronic reprint of the original article. This reprint may differ from the original in pagination and typographic detail.

\author{
Author(s): Koivisto, Hannu; Komppula, Jani; Kronholm, Risto; Tarvainen, Olli; Toivanen, Ville; \\ Lamy, T.; Angot, J.; Delahaye, P.; Maunoury, L.; Galata, A.; Patti, G.; Standylo, L.; \\ Steczkiewicz, O.; Choinski, J.
}

Title: Ionization efficiency studies with charge breeder and conventional electron cyclotron resonance ion source

Year: $\quad 2014$

Version:

Please cite the original version:

Koivisto, H., Komppula, J., Kronholm, R., Tarvainen, O., Toivanen, V., Lamy, T., Angot, J., Delahaye, P., Maunoury, L., Galata, A., Patti, G., Standylo, L., Steczkiewicz, O., \& Choinski, J. (2014). Ionization efficiency studies with charge breeder and conventional electron cyclotron resonance ion source. Review of Scientific Instruments, 85(2), Article 4854215. https://doi.org/10.1063/1.4854215

All material supplied via JYX is protected by copyright and other intellectual property rights, and duplication or sale of all or part of any of the repository collections is not permitted, except that material may be duplicated by you for your research use or educational purposes in electronic or print form. You must obtain permission for any other use. Electronic or print copies may not be offered, whether for sale or otherwise to anyone who is not an authorised user. 


\section{AP $\mid \begin{aligned} & \text { Review of } \\ & \text { Scientific Instruments }\end{aligned}$}

\section{lonization efficiency studies with charge breeder and conventional electron cyclotron resonance ion sourcea)}

H. Koivisto, O. Tarvainen, V. Toivanen, J. Komppula, R. Kronholm, T. Lamy, J. Angot, P. Delahaye, L. Maunoury , A. Galata, G. Patti, L. Standylo, O. Steczkiewicz, and J. Choinski

Citation: Review of Scientific Instruments 85, 02B917 (2014); doi: 10.1063/1.4854215

View online: http://dx.doi.org/10.1063/1.4854215

View Table of Contents: http://scitation.aip.org/content/aip/journal/rsi/85/2?ver=pdfcov

Published by the AIP Publishing

\section{Articles you may be interested in}

Optimization of a hot-cavity type resonant ionization laser ion source

Rev. Sci. Instrum. 87, 02B701 (2016); 10.1063/1.4931618

Operational experience with the Argonne National Laboratory Californium Rare Ion Breeder Upgrade facility and electron cyclotron resonance charge breedera)

Rev. Sci. Instrum. 85, 02B903 (2014); 10.1063/1.4826329

Improved charge breeding efficiency of light ions with an electron cyclotron resonance ion source Rev. Sci. Instrum. 83, 113303 (2012); 10.1063/1.4768533

Production of charged (singly and multiply) phosphorous beams with electron cyclotron resonance ion source Rev. Sci. Instrum. 77, 03 A324 (2006); 10.1063/1.2166433

Efficiency and transient time studies of an electron cyclotron resonance ion source for radioactive ion beam production at ISAC/TRIUMF

Rev. Sci. Instrum. 71, 946 (2000); 10.1063/1.1150353

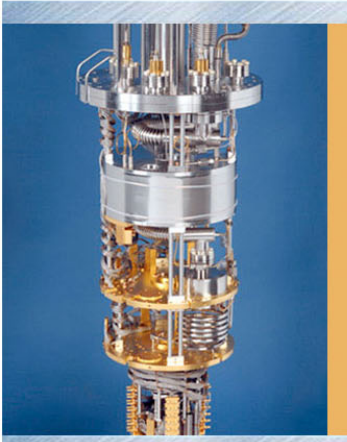

\section{JAN I S}

Janis Dilution Refrigerators \& Helium-3 Cryostats for Sub-Kelvin SPM

Click here for more info www.janis.com/UHV-ULT-SPM.aspx 


\title{
Ionization efficiency studies with charge breeder and conventional electron cyclotron resonance ion source ${ }^{a)}$
}

\author{
H. Koivisto, ${ }^{1, b)}$ O. Tarvainen, ${ }^{1}$ V. Toivanen, ${ }^{1}$ J. Komppula, ${ }^{1}$ R. Kronholm,${ }^{1}$ T. Lamy, ${ }^{2}$ \\ J. Angot, ${ }^{2}$ P. Delahaye,${ }^{3}$ L. Maunoury, ${ }^{3}$ A. Galata, ${ }^{4}$ G. Patti, ${ }^{5}$ L. Standylo, ${ }^{6}$ \\ O. Steczkiewicz, ${ }^{6}$ and J. Choinski6 ${ }^{6}$ \\ ${ }^{1}$ Department of Physics, University of Jyväskylä (JYFL), Jyväskylä, Finland \\ ${ }^{2}$ LPSC, Université Joseph Fourier Grenoble 1, Grenoble INP, 53 rue des martyrs, 38026 Grenoble Cedex, \\ France \\ ${ }^{3}$ GANIL, CEA/DSM-CNRS/IN2P3, Caen Cedex 05, France \\ ${ }^{4}$ INFN-Laboratori Nazionali di Legnaro, Legnaro, Padova, Italy \\ ${ }^{5}$ INFN-Laboratori Nazionali del Sud, Catania, Italy \\ ${ }^{6}$ Heavy Ion Laboratory, University of Warsaw, Warsaw, Poland
}

(Presented 12 September 2013; received 5 September 2013; accepted 11 November 2013; published online 2 January 2014)

\begin{abstract}
Radioactive Ion Beams play an increasingly important role in several European research facility programs such as SPES, SPIRAL1 Upgrade, and SPIRAL2, but even more for those such as EURISOL. Although remarkable advances of ECRIS charge breeders (CBs) have been achieved, further studies are needed to gain insight on the physics of the charge breeding process. The fundamental plasma processes of charge breeders are studied in the frame of the European collaboration project, EMILIE, for optimizing the charge breeding. Important information on the charge breeding can be obtained by conducting similar experiments using the gas mixing and 2-frequency heating techniques with a conventional JYFL $14 \mathrm{GHz}$ ECRIS and the LPSC-PHOENIX charge breeder. The first experiments were carried out with noble gases and they revealed, for example, that the effects of the gas mixing and 2 -frequency heating on the production of high charge states appear to be additive for the conventional ECRIS. The results also indicate that at least in the case of noble gases the differences between the conventional ECRIS and the charge breeder cause only minor impact on the production efficiency of ion beams. () 2014 AIP Publishing LLC. [http://dx.doi.org/10.1063/1.4854215]
\end{abstract}

\section{INTRODUCTION}

Charge breeders $(\mathrm{CBs})^{1-3}$ can be considered as key components in the operation of Radioactive Ion Beam (RIB) facilities using ISOL technique. The most important parameters of the breeding process are the accessible charge state, purity of the beam, breeding time, and efficiency. This work concentrates on the production efficiency of highly charged noble gas ion beams. The experiments were carried out with the JYFL 14.1 GHz ECR ion source and the $14.5 \mathrm{GHz}$ Phoenix charge breeder. These two ion sources have approximately the same plasma heating frequency and the same plasma volume. The main differences between these ion sources are caused by the element injection scheme.

The injection scheme differs between a normal ECRIS and a charge breeder. In the case of the charge breeder the element of interest is injected as a 1+ ion, whilst in the case of the ECRIS the element is fed into the ion source as neutral gas. The $1+$ beam is injected axially (on axis and along the magnetic field), which generates two significant operational deviations compared to normal ECRISs. The axial 1+ injection (1) prohibits the use of the bias disc and (2) causes modifications to iron configuration, which results in lower injec-

\footnotetext{
${ }^{a)}$ Contributed paper, published as part of the Proceedings of the 15th International Conference on Ion Sources, Chiba, Japan, September 2013.

b) Author to whom correspondence should be addressed. Electronic mail: hannu.koivisto@phys.jyu.fi.
}

tion magnetic field $\mathrm{B}_{\mathrm{inj}}$. In the case of ECRIS the magnetic field configuration and the bias disc both have a strong impact on the intensities and production efficiencies of highly charged ion beams. More information about their effect can be found from Refs. 4 and 5 and references therein. The motivation of this study was to compare the production efficiency of aforementioned conventional ECRIS and charge breeder and to quantify parameters affecting the production efficiency of highly charged ion beams.

\section{EXPERIMENTS WITH CONVENTIONAL ECRIS}

The first set of production efficiency measurements were performed with the JYFL $14 \mathrm{GHz}$ ECRIS. ${ }^{6}$ Here the production efficiency is the ratio of neutral particles injected into the ion source and measured as a beam following the $\mathrm{m} / \mathrm{q}$ separation. Calibration curves for each gas were defined in order to know the feed rate of the gas as a function of plasma chamber partial pressure. The error concerning the consumption rate is estimated to be about $\pm 10 \%$ for plasma chamber pressure of $5 \times 10^{-7}$ mbar or higher. In the case of the lowest feed rate the error was estimated to be $\pm 30 \%$. The background pressure $\left(9.6 \times 10^{-8}\right.$ mbar) was taken into account in all results presented. The pressure values have been measured without plasma.

The main objective of the experiments with the JYFL $14 \mathrm{GHz}$ ECRIS was to study systematically the effect of gas 
mixing $^{7}$ and 2 -frequency heating techniques ${ }^{8}$ on the production efficiencies of highly charged ion beams. The ion source was tuned using four different operation combinations: (1) gas of interest without mixing gas nor 2-frequency heating, (2) gas of interest with oxygen as a mixing gas, (3) gas of interest with 2-frequency heating, and (4) gas of interest with oxygen as a mixing gas and 2-frequency heating. The parameters (feed rates, B-field, and bias disk) were optimized for the production of maximum intensities of ${ }^{40} \mathrm{Ar}^{12+},{ }^{84} \mathrm{Kr}^{20+}$, and ${ }^{136} \mathrm{Xe}^{24+}$ ion beams. In all cases the total $\mu$ w-power of $600 \mathrm{~W}$ and extraction voltage of $12 \mathrm{kV}$ was used. After the optimization the gas feed rate and the intensities of different charge states were measured enabling one to calculate the production efficiency of each charge state. It should be noted that the production efficiency includes the ionization efficiency and the beam transport between the ion source and the Faraday cup downstream from the m/q-separation. The beam formation (extraction) was not space charge limited and consequently it is assumed that the changes in production efficiency are mainly due to the changes in ionization efficiency.

\section{A. Production efficiencies of different gases}

Figure 1 shows the production efficiencies obtained with $\mathrm{Kr}$ under different operational combinations. All charge states were not measured due to the limitations in mass separation and beam optics. A significant improvement in the production efficiency of highly charged ion beams was obtained with the gas mixing. The technique allows to decrease the feed rate of the heavier element and yet, improves the intensities of highly charged ions. As a result, their production efficiency has improved by a factor of 5-10. The figure shows also that the gas mixing makes it possible to shift the charge state distribution (CSD) towards higher charge states. Similar efficiency tendencies were observed with $\mathrm{Ar}$ and Xe.

In the 2-frequency heating experiments the power of the secondary frequency $(11.56 \mathrm{GHz})$ was $60 \mathrm{~W}$ while the total power was kept at $600 \mathrm{~W}$. Relatively long operational experience and dedicated experiments ${ }^{9}$ have shown that this frequency selection leads to stable operation and good performance. According to the results, the 2-frequency heating has a positive impact only on the high charge states $\left(\geq \mathrm{Ar}^{11+}\right.$,

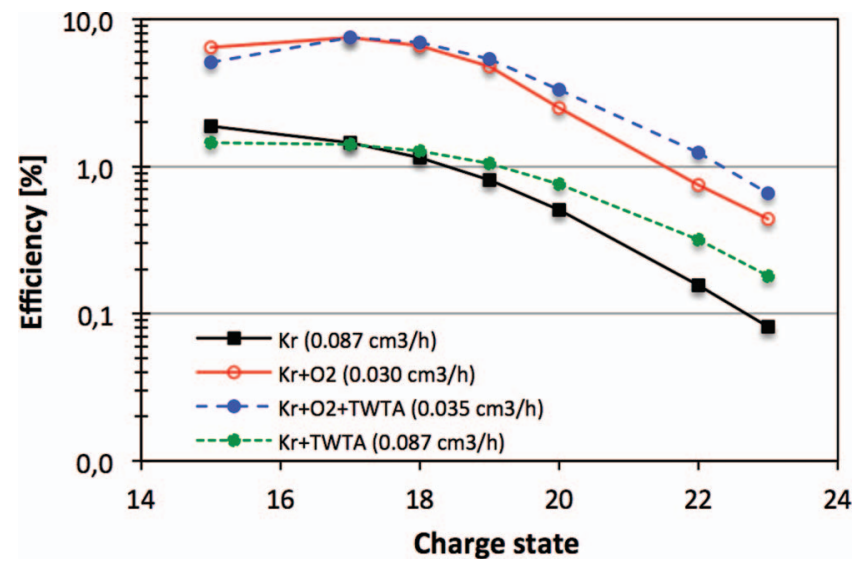

FIG. 1. Production efficiencies of $\mathrm{Kr}$ ion beams with and without the use of gas mixing and 2-frequency heating.

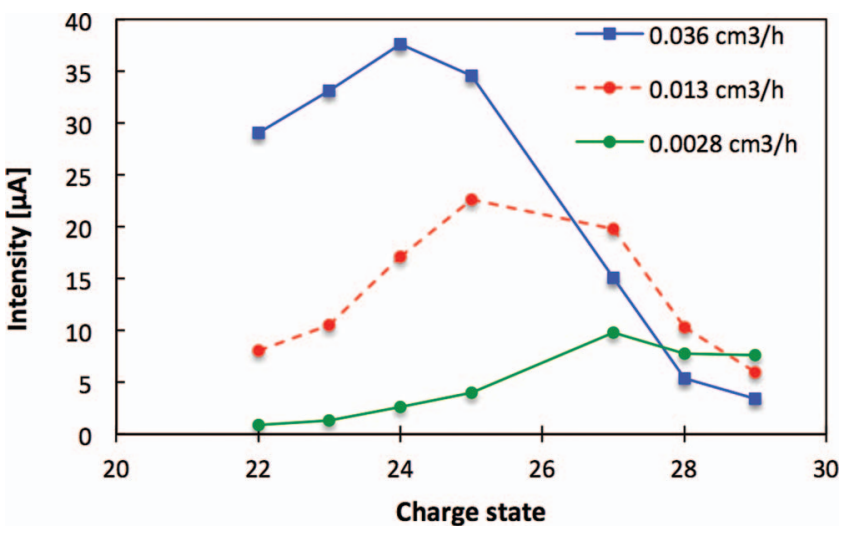

FIG. 2. $\mathrm{Xe}^{22-29+}$ currents with different gas feed rates.

$\geq \mathrm{Kr}^{19+}$, and $\geq \mathrm{Xe}^{25+}$ ). The effect increased with the charge state and approximately doubled the production efficiency of $\mathrm{Ar}^{14+}, \mathrm{Kr}^{23+}$, and $\mathrm{Xe}^{29+}$ ion beams.

The results indicate that the improvements due to gas mixing and 2-frequency heating are additive, which suggests that these techniques improve the ion source performance via different mechanisms. It is assumed that the gas mixing affects mainly the ion population via cooling effect (see Ref. 7) and the 2-frequency technique heats the electrons and consequently can affect directly the density of electron population and their energy distribution. It is also possible that 2 -frequency heating modifies the plasma potential structure and therefore has an indirect effect on the ion confinement. ${ }^{10}$ Neither of the techniques was observed to have a clear impact on the width of the CSD.

\section{B. The effect of low gas feed rate}

In the previous experiments the ion beam intensities were maximized without restricting the gas feed rates. As a next step the xenon feed rate was decreased significantly below the value corresponding to the highest $\mathrm{Xe}^{24+}$ intensity. This experiment was carried out using three xenon neutral feed rates, i.e., $0.036 \mathrm{~cm}^{3} / \mathrm{h}(40 \mathrm{p} \mu \mathrm{A}), 0.013 \mathrm{~cm}^{3} / \mathrm{h}(15 \mathrm{p} \mu \mathrm{A})$, and $0.0028 \mathrm{~cm}^{3} / \mathrm{h}(3.1 \mathrm{p} \mu \mathrm{A})$. The corresponding particle-feed rates are listed in the parenthesis. The value of $0.036 \mathrm{~cm}^{3} / \mathrm{h}$ corresponds to the highest $\mathrm{Xe}^{24+}$ ion beam intensity and the lowest value is close to the typical $1+$ beam intensity fed into the CB. In this study the ion source was carefully tuned to maximize the intensity of $\mathrm{Xe}^{29+}$ ion beam. As Fig. 2 shows the intensity of charge states $<27+$ decreased substantially with the lower feed rate whilst the intensities of the charge states $>27+$ increased. The production efficiencies of very high charge states increased dramatically - for example, by a factor of about 20 in the case of $\mathrm{Xe}^{29+}$ as demonstrated in Fig. 3.

During the experiment the 2-frequency heating was ON and oxygen was used as a mixing gas. The partial pressure of oxygen was $5.4 \times 10^{-7} \mathrm{mbar}(13 \%), 5.0 \times 10^{-7}(6 \%) \mathrm{mbar}$, and $3.3 \times 10^{-7} \mathrm{mbar}(1.8 \%)$, respectively. The mixing ratio of xenon is shown in the parenthesis (values include the gas calibration factor). The lower mixing ratio of the heavier element will increase the probability of (Coulomb) collisions with lighter element resulting in more efficient cooling 


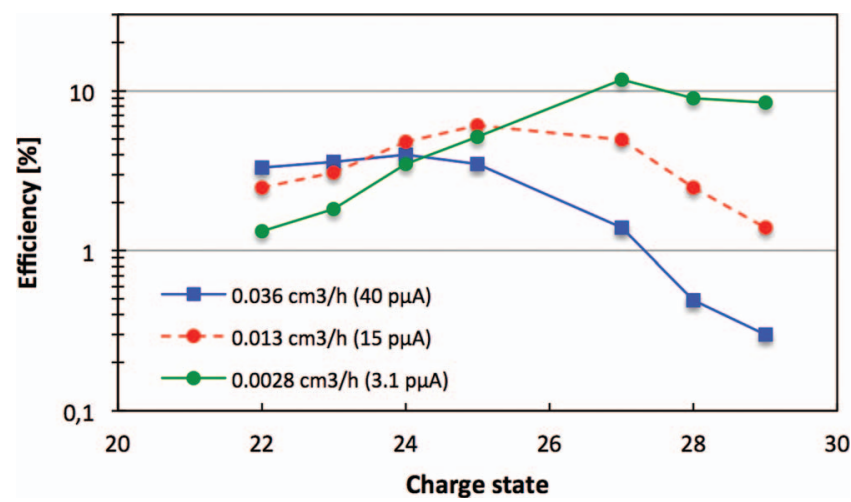

FIG. 3. Production efficiencies of $\mathrm{Xe}^{22-29+}$ ion beams with different gas feed rates.

of the heavier element while lower total neutral injection can decrease the charge exchange rate. Both have a positive impact on the production of the highest charge states, which are in accordance with observations.

\section{EXPERIMENTS WITH CHARGE BREEDER}

The second set of the experiments was performed with the Phoenix charge breeder ${ }^{11,12}$ on the LPSC test stand. The $1+$ beam injected into the breeder was produced with the COMIC ion source. ${ }^{13}$ The main emphasis was on the 2frequency heating studies due to the high background pressure $\left(\approx 3 \times 10^{-7}\right.$ mbar $)$ of the $\mathrm{CB}$ making it difficult to resolve the gas mixing effect. The partial neutral pressure caused by the gas flow from COMIC was $\approx 1 \times 10^{-7}$ mbar. Consequently, the element of interest was fed into the booster as neutral gas and $1+$ ions simultaneously. The resulting background current was defined by switching OFF the $1+$ injection and has been taken into account when calculating the efficiencies. This background current was in some cases as high as tens of per cents of the charge bred current.

Figure 4 shows the production efficiency of the Phoenix $\mathrm{CB}$ as a function of $\mathrm{Ar}^{+}$ion beam intensity at constant $\mathrm{CB}$ settings. The 2-frequency heating was used and small amount of oxygen was fed into the breeder, which increased the efficiency. With the proper $\mathrm{CB}$ settings, the $\mathrm{Ar}^{11+}$ produc-

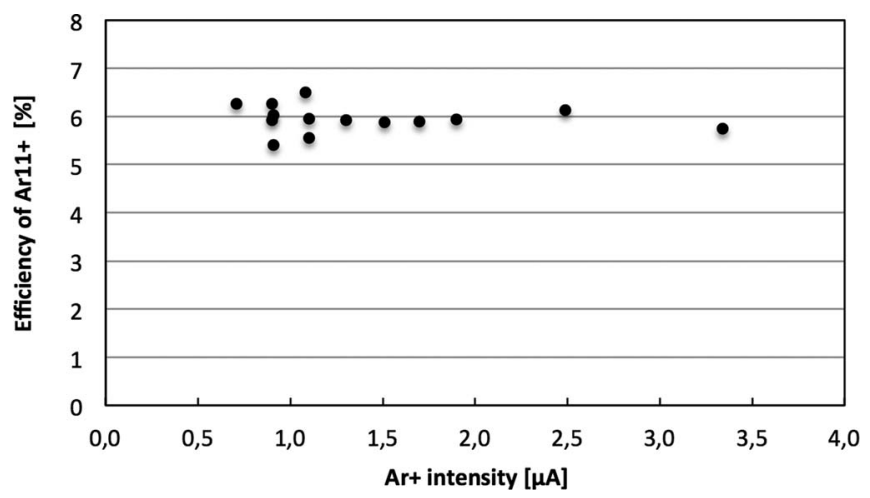

FIG. 4. Production efficiency of $\mathrm{Ar}^{11+}$ ion beams as a function of $\mathrm{Ar}^{+}$beam intensity injected from COMIC into Phoenix CB.

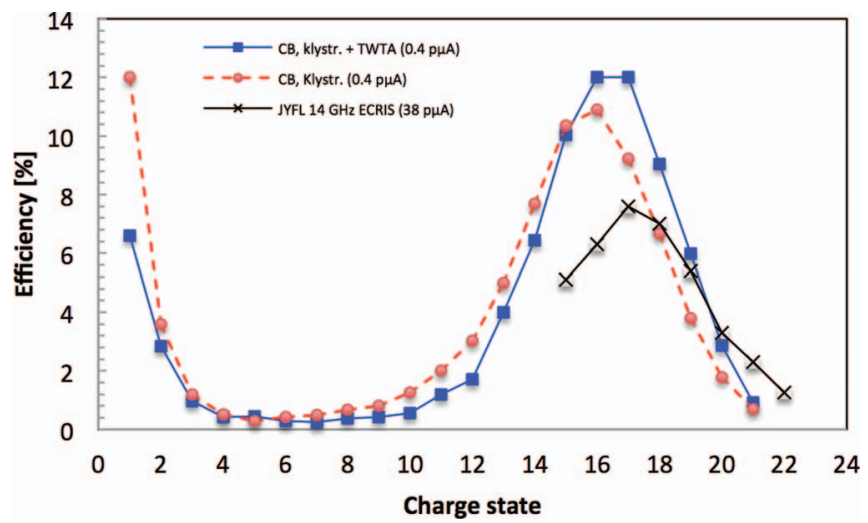

FIG. 5. Effect of 2-frequency heating on the intensities of krypton ion beams.

tion efficiency is constant over a wide range of $1+$ beam intensities.

In the following experiments ${ }^{40} \mathrm{Ar}^{+}$or ${ }^{78} \mathrm{Kr}^{+}$ion beams were fed into the CB. The breeder was optimized for high charge states of argon or krypton and the intensities of all charge states were subsequently measured. The optimization was done with and without 2-frequency heating $(14.5 \mathrm{GHz}$ klystron + adjustable frequency TWTA). Figure 5 shows a typical result with krypton (tuned for $\mathrm{Kr}^{21+}$ ). In this case, $414 \mathrm{nA}$ of $\mathrm{Kr}^{+}$beam was injected into the charge breeder. In both cases (with/without 2-frequency heating) the total microwave power of $600 \mathrm{~W}$ was used. A total production efficiency of about $80 \%$ was measured with and without 2 -frequency heating (charge states from $1+$ to $21+$ ). This is an indication of very efficient capture of the $1+$ beam (see also Ref. 3). As a comparison, the result with the JYFL $14 \mathrm{GHz}$ ECRIS is presented in the same figure. The production efficiency with the LPSC CB is slightly higher than with the JYFL $14 \mathrm{GHz}$ ECRIS but in all three cases the spectrum is peaked on $\mathrm{Kr}^{16-17+}$ ion beam.

The effect of injection scheme $(1+$ versus neutral injection) on the charge state distribution was studied with the Phoenix charge breeder. The $\mathrm{CB}$ was optimized for $\mathrm{Kr}^{21+}$ with the gas mixing and 2-frequency technique. As a first step, the total krypton beam intensity, produced from the neutral background flow from COMIC (1+ beam was OFF), was measured to be about $100 \mathrm{pnA}$. The aforementioned intensity included charge states from $1+$ to $21+$. The intensity of some charge states were estimated from the CSD because of the overlapping caused by residual components (mainly oxygen and nitrogen). As a second step the $1+$ injection (414 nA) was turned $\mathrm{ON}$ and aforementioned CSD was measured with and without the 2-frequency heating. The normalized efficiency distributions are shown in Fig. 6. The JYFL result, with the gas mixing and 2-frequency heating, is shown for comparison.

As figure shows a fairly similar normalized production efficiency distribution for the highest charge state $(\mathrm{q}=14-21)$ is obtained in all four cases. The figure also shows the positive effect of 2-frequency heating on the highest charge states. Furthermore the figure shows that high intensity is measured for single charged krypton ion beam in the case of $1+$ injection. This indicates that a fraction of the $1+$ ions (around 


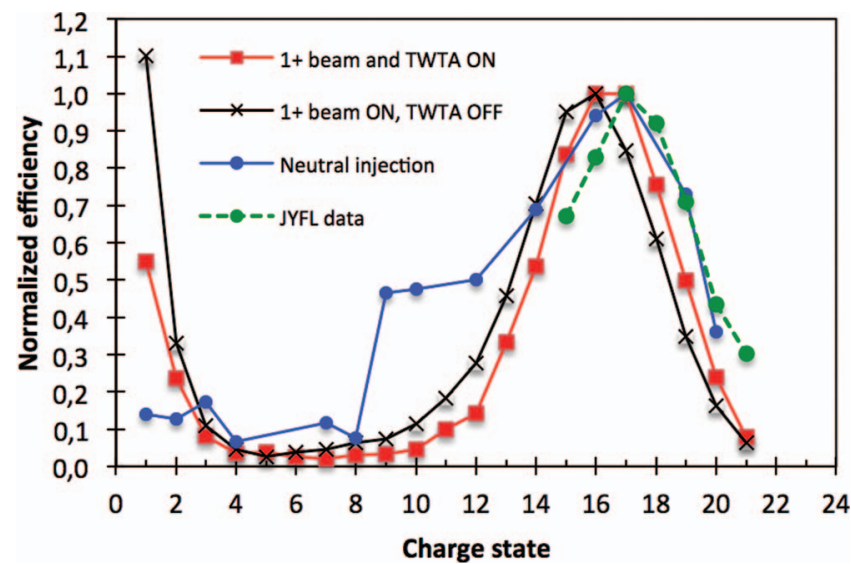

FIG. 6. Normalized production efficiencies of krypton ion beams with the LPSC charge breeder. The efficiencies are presented for neutral injection and for charge bred beams with and without the 2 -frequency heating. In each case, the highest efficiencies of high charge states $(q=16$ or 17) have been normalized to the value of 1 . The JYFL result is shown for the comparison.

$10 \%$ ) propagate through the plasma without being captured through Coulomb collisions and further ionized.

Fig. 7 shows the production efficiencies of $\mathrm{Ar}^{11+}$ ion beams as a function of the TWTA frequency and power. A fixed klystron power of $250 \mathrm{~W}$ was used for the given data set. The gain of the TWTA was set to a constant value for the frequency sweep, which caused the forward power to fluctuate with varying load. The red line in Fig. 7 (TWTA tuning) corresponds to the efficiency in single frequency heating mode with $250 \mathrm{~W}$ klystron power.

In the lower part of Fig. 7 the red line corresponds to the efficiency achieved in single frequency mode with matching the klystron power to the total power in 2-frequency mode. The 2-frequency heating benefits the production of highly
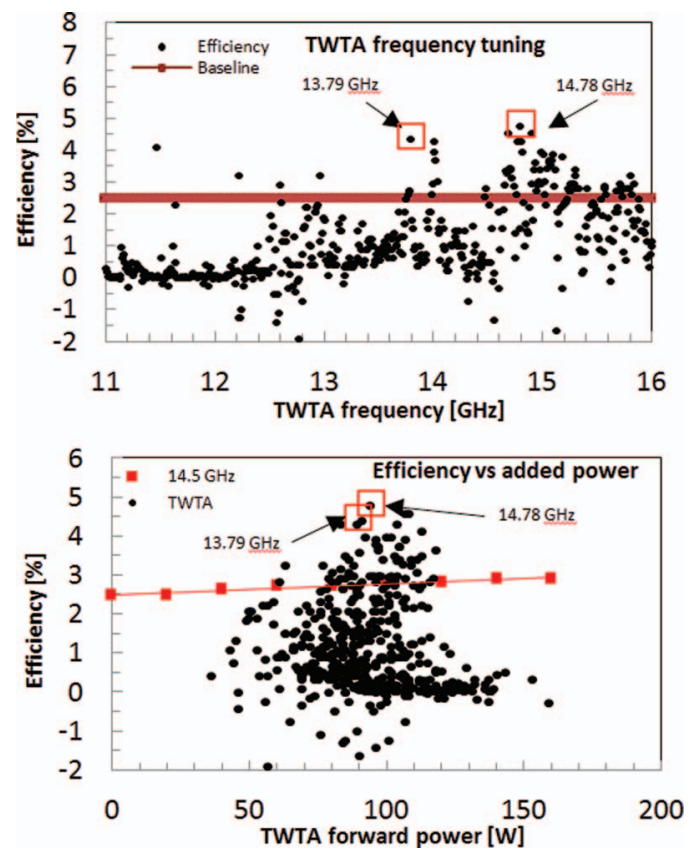

FIG. 7. Production efficiencies of $\mathrm{Ar}^{11+}$ ion beams as a function of TWTA frequency and power. Red baseline corresponds to the results when the plasma was heated using the klystron alone with the equivalent total power. charged ions in the case of those data points above the red line. It should be emphasized that the beneficial effect is obtained only with the proper selection of secondary frequency as is demonstrated by Fig. 7. It is also important to recognize that at some secondary frequencies the effect on breeding efficiency was negative. This means that $1+$ injection resulted to a total $\mathrm{Ar}^{11+}$ current lower than the background.

\section{DISCUSSION}

The experiments with the conventional JYFL $14 \mathrm{GHz}$ ECRIS and the LPSC Phoenix charge breeder show that, at least in the case of krypton, fairly similar charge state efficiency distribution is achieved (see Fig. 6). However, it is possible that CSD will slightly move towards higher charge states if lower krypton feed would have been used during the JYFL experiment $(\approx 1 \mathrm{p} \mu \mathrm{A}$ instead of $40 \mathrm{p} \mu \mathrm{A})$. Fig. 3 supports this interpretation. The results also indicate that the lack of bias disk and considerably lower B field in the injection (1.2 T versus $2.2 \mathrm{~T}$ ) did not have very strong impact on the production efficiency distribution in the case of the noble gases.

As a next step, similar experiments will be carried out using solid elements. These shall $a$ priori be more sensitive to potential differences between the two methods, as in charge breeders and for these elements a direct capture into the plasma is required. The use of solid elements enables a more detailed study of the $1+$ capture process. It is important to measure the fraction of the $1+$ beam that seems to propagate through the plasma. Such study can reveal new information about the capture process, 2-frequency heating mechanism, mean-free path of ions, and even about the plasma density.

\section{ACKNOWLEDGMENTS}

This work has been supported by the EU 7th framework program "Integrating Activities - Transnational Access," Project No.: 262010 (ENSAR), by the ERA-NET NuPNET and by the Academy of Finland under Finnish Centre of Excellence Programme 2012-2017 (Nuclear and Accelerator Based Physics Research at JYFL).

${ }^{1}$ T. Lamy, R. Geller, P. Sortais, and T. Thuillier, Rev. Sci. Instrum. 77, 03B101 (2006).

${ }^{2}$ P. Delahaye, O. Kester, C. Barton, T. Lamy, M. Marie-Jeanne, and F. Wenander, Eur. Phys. J. A 46, 421 (2010).

${ }^{3}$ R. Vondrasek et al., Rev. Sci. Instrum. 83, 02 A913 (2012).

${ }^{4}$ A. Girard, D. Hitz, G. Melin and K. Serebrennikov, Rev. Sci. Instrum. 75(5), 1381 (2004).

${ }^{5}$ D. Leitner, C. M. Lyneis, D. S. Todd, O. Tarvainen, Nucl. Instrum. Methods Phys. Res. B 264, 149-155 (2007).

${ }^{6} \mathrm{H}$. Koivisto et al., Nucl. Instrum. Methods Phys. Res. B 174, 379 (2001).

${ }^{7}$ A. G. Drentje, Rev. Sci. Instrum. 74(5) 2631 (2003) and references therein.

${ }^{8} \mathrm{Z}$. Q. Xie and C. M. Lyneis, Proceedings of the 12th International Workshop on ECR Ion Sources, Riken (Report: INS-J-182, RIKEN, Tokyo, Japan, 1995), p. 24.

${ }^{9}$ O. Tarvainen, P. Suominen, T. Roppponen, and H. Koivisto, Rev. Sci. Instrum. 77, 03A309 (2006).

${ }^{10}$ R. C. Vondrasek, R. Scott, and R. C. Pardo, Rev. Sci. Instrum. 77, 03 A337 (2006).

${ }^{11}$ P. Sortais, J. F. Bruandet, J. L. Bouly, N. Chauvin, J. C. Curdy, R. Geller, T. Lamy, P. Sole, and J. L. Vieux-Rochaz, Rev. Sci. Instrum. 71, 617 (2000).

${ }^{12}$ T. Lamy, J. Angot, P. Sortais, T. Thuillier, and A. Galata, Rev. Sci. Instrum. 83, 02 A909 (2012).

${ }^{13}$ P. Sortais, T. Lamy, J. Médard, J. Angot, L. Latrasse, and T. Thuillier, Rev. Sci. Instrum. 81, 02B314 (2010). 